\title{
Ingresos y felicidad: paradoja de Easterlin en Colombia*
}

\author{
[Versión en Castellano] \\ Income and Happiness: Easterlin Paradox in Colombia \\ Renda e Felicidade: Paradoxo de Easterlin na Colômbia
}

Recibido 8 de abril, 2020. Aceptado 7 de octubre, 2020.

Héctor-Alberto Botello-Peñaloza**
https://orcid.org/0000-0002-7795-2590
Colombia
Isaac Guerrero-Rincón***
https://orcid.org/0000-0001-5794- 2742

Colombia

, Para citar este artículo:

Botello-Peñaloza, Héctor-Alberto;

Guerrero-Rincón, Isaac (2021).

Ingresos y felicidad: paradoja de

Easterlin en Colombia.

Ánfora, 28(50), 275-294.

https://doi.org/10.30854/anfv28.n50.2021.696

Universidad Autónoma de

Manizales. ISSN 0121-6538/

e-ISSN 2248-6941.

CC BY-NC-SA 4.0

\section{Resumen}

Objetivo: este trabajo busca comprobar la relación entre ingreso y felicidad en Colombia, haciendo especial énfasis en la linealidad de esta relación conocida en la con base en los datos de la Encuesta de Calidad de Vida 2017 se constató si la población colombiana se adapta a la paradoja; es decir, si el ingreso monetario influye positivamente en el bienestar subjetivo revelado. Resultados: el modelo ordinal propuesto evidencia que literatura como la "paradoja de Easterlin". Metodología:

\footnotetext{
* El origen del artículo de investigación surge del Grupo de Investigación sobre Desarrollo Regional y Planeación Territorial. Los autores declaran que no hubo conflicto de intereses en la ejecución del proyecto de investigación.

** Magister ciencias económicas. Economista. Universidad Industrial de Santander. Correo electrónico: hbotellop@unal.edu.co

**** Magíster en Economía, Universidad Nacional de Colombia, Bogotá, Colombia. Profesor de la Universidad Industrial de Santander, Bucaramanga, Santander, Colombia. Miembro del Grupo de Investigación sobre Desarrollo Regional y Ordenamiento Territorial.Correo electrónico: iguerrin@uis.edu.co
} 
el ingreso es una variable determinante de la felicidad, pero es secundaria frente a otras como la percepción del bienestar en salud, seguridad y trabajo. Estos resultados están en línea con los planteados en la paradoja y plantean desafíos de los planes de desarrollo donde un contexto integral de bienestar es el que logra incrementar la calidad de vida de los habitantes. Conclusiones: la felicidad revelada por los individuos es de tipo subjetivo, por lo tanto, puede estar influida por aspectos coyunturales al momento de la encuesta.

Palabras-clave: Economía de la felicidad; Paradoja de Easterlin; Modelo ordinal; Ingreso; Colombia.

\section{Abstract}

Objective: this research aims to verify the relationship between income and happiness in Colombia, with special emphasis on the linearity of this relationship known in literature as the "Easterlin paradox". Methodology: based on data from Quality of Life Survey of 2017, it was investigated into whether the Colombian population conforms to the paradox, that is, whether monetary income positively influences the subjective well-being revealed. Results: the proposed proportional odds model shows that income is a determining variable of happiness, but it is secondary to others such as the perception of well-being in health, safety and work. These results coincide with those raised in the paradox, and challenge development plans in which a comprehensive context of well-being increases the quality of life of the inhabitants. Conclusions: the happiness revealed by individuals is subjective in nature, therefore, it may be influenced by conjunctural aspects at the time of the survey.

Keywords: Economics of happiness; Easterlin paradox; Proportional odds model; Income; Colombia. 


\section{Resumo}

Objetivo: este trabalho busca verificar a relação entre renda e felicidade na Colômbia, com ênfase especial na linearidade dessa relação conhecida na literatura como o "paradoxo de Easterlin". Metodologia: com base em dados da Pesquisa de Qualidade de Vida de 2017 conseguiu se descobrir se a população colombiana se adapta ao paradoxo; ou seja, se o rendimento monetário influencia positivamente o bem-estar subjetivo revelado. Resultados: o modelo ordinal proposto mostra que a renda é uma variável determinante da felicidade, mas é secundária a outras como a percepção de bem-estar na saúde, segurança e trabalho. Esses resultados estão em consonância com os levantados no paradoxo e colocam desafios dos planos de desenvolvimento onde um contexto abrangente de bem-estar é aquele que consegue aumentar a qualidade de vida dos habitantes. Conclusões: a felicidade revelada pelos indivíduos é subjetiva por natureza, portanto pode ser influenciada por aspectos conjunturais no momento da pesquisa.

Palabras-chave: Economia da felicidade; Paradoxo da Páscoa; Modelo ordinal; Entrada; Colômbia. 


\section{Introducción}

La Encuesta de Valores Mundiales de 2006 (WVS) muestra que Colombia es el segundo país más feliz del mundo, con un ingreso per cápita promedio de 6000 dólares al año. La situación de violencia, desigualdad y criminalidad del país hacen de Colombia un caso curioso para explicar el porqué de esta situación. Una posible explicación de esto es la denominada paradoja de Easterlin.

De acuerdo con Veenhoven (2007), la felicidad de los individuos conlleva información importante sobre la calidad de los modelos de desarrollo impulsados por los gobiernos. Los estudios sobre la economía de la felicidad son importantes, ya que el bienestar de los individuos retroalimenta positivamente diversas dimensiones de sus vidas, por ejemplo, su productividad y la convivencia social (Torrecilla, 2005; Botello y Ríos, 2014). Por lo tanto, la felicidad es un componente con significativas connotaciones sociales, políticas y económicas que se deben ponderar en el modelo de desarrollo actual.

Los resultados de esta investigación ayudarían al gobierno a promover o reforzar políticas económicas que impulsen el bienestar de los habitantes. En consecuencia, existe una necesidad de investigar los determinantes de la felicidad de los individuos; sin embargo hay evidencia restringida empírica robusta en Colombia para resolver esta teoría. Este estudio, entonces, ofrece validar dos hipótesis: la principal, es que el ingreso genera un efecto positivo en la felicidad de las personas, pero no es el componente más importante. En segundo lugar, busca comprobar la inferencia que, a partir de cierto umbral de aumento del ingreso, este ya no ejerce una influencia en la felicidad de las personas.

Este trabajo contribuye a la literatura en dos formas. La primera, porque utiliza una base amplia de microdatos de individuos y sus niveles de satisfacción con varias dimensiones de su vida. Además, se usa un modelo ordinal probit para determinar los diferentes niveles de felicidad y no modelos binomiales. La utilización de este modelo permitirá comparar los resultados con otros encontrados en la literatura de la felicidad en Latinoamérica. Finalmente, el modelo utilizado sirve para cardinalizar los factores que ponderan los colombianos al considerar sus niveles de satisfacción subjetiva, tales como la salud, el trabajo y la seguridad.

El modelo ordinal propuesto evidencia que el ingreso es una variable determinante de la felicidad, pero es secundaria frente a otras como la percepción de salud, seguridad y trabajo: los resultados están en línea con los planteados en la paradoja y en la evidencia internacional. El efecto marginal de cada cuantil de ingreso muestra que el efecto del ingreso sobre la felicidad es marginalmente lineal, es decir, que no se cumple la hipótesis de que, a partir de cierto umbral de ingreso, este ya no ejerce una influencia en la felicidad de las personas. 
Los limitantes del presente estudio se basan en la fuente de información, dado que la felicidad revelada por los individuos es de tipo subjetivo, puede estar influida por aspectos coyunturales al momento de la encuesta. Igualmente, las extensiones del presente estudio pueden estar enfocados al análisis longitudinal del individuo y cómo cambian sus percepciones frente a cambios de la política pública aplicada a su contexto individual, familiar y de riqueza.

\section{Marco teórico}

Aunque las cavilaciones sobre las relaciones entre el ingreso y la felicidad se habían dado en debates filosóficos desde el siglo XVI, fue en 1974 cuando el economista Richard Easterlin (1974) realizó los primeros estudios empíricos, con los que buscó cuantificar el aporte del ingreso en el bienestar de los individuos. Esta línea de investigación surgió dada la atención central que se tenían en temas como el crecimiento económico, pero se descuidaba que el bienestar de las personas debería ser el principal objetivo de las economías.

En el modelo del consumidor que enseña la microeconomía tradicional, una mayor cantidad de ingreso expande la frontera de posibilidades de consumo del individuo al afectar la restricción presupuestaria. Este movimiento incrementa la utilidad experimentada por el individuo. Entonces, un mayor ingreso impacta positivamente el bienestar económico. En este sentido, Easterlin (1974) encontró que si bien la felicidad crece con el ingreso, esta relación cesa de existir después de cierto umbral; en pocas palabras, "el dinero no compra felicidad".

Sus explicaciones a este fenómeno contra-intuitivo a la microeconomía tradicional fueron dos. La primera, la gente se adapta a su ingreso y a las posibilidades que este dispone. Esta asimilación neutraliza el efecto de los mayores ingresos dado que las expectativas de consumo y disfrute aumentan al mismo ritmo y mantiene la felicidad al mismo nivel. La segunda, la gente compara sus ingresos actuales con su experiencia previa o su entorno y de allí surgen, mediante valoraciones subjetivas, su definición de felicidad (Frank, 1985). En consecuencia, e bienestar individual es una experiencia personal que depende considerablemente del entorno social. El anterior resultado impulsó un conjunto de investigaciones orientadas a evaluar la histórica correspondencia entre ingreso y felicidad.

Revisión de literatura

La felicidad es una evaluación subjetiva que realiza una persona de sus dimensiones cognitivas y afectivas (Diener et al., 1995). La sensación de libertad y el control sobre la misma se asocia con una autoevaluación positiva de esta situación (Reich y Diener, 1994). Al encontrarse en una sociedad imbuida por el bienestar material, el control se relaciona con la posición de bienes y servicios. 
De aquí la justificación de abordar los determinantes de la felicidad desde un punto de vista de la riqueza (Borrero et al., 2013). Aquí entra el papel de la paradoja de Easterlin, donde se han planteado dos aproximaciones metodológicas para abordarla.

El primero es a través de datos macroeconómicos cómo el crecimiento del PIB per cápita (u otras medidas) afectan el nivel medio de felicidad reportado. Entre los resultados más recientes están en Easterlin (1995), Oishi y Kesebir (2015) y Mikucka, Sarracino y Dubrow (2017). Estos autores hallaron evidencia que el crecimiento económico incrementa la felicidad en promedio, pero, se requiere que ésta se acompañe de una reducción de la desigualdad. Con microdatos, en los países desarrollados donde las encuestas de ingresos se combinan con cuestionarios de percepción, han sido abundantes los trabajos que han validado la paradoja de Easterlin, aunque con resultados ambivalentes debido a los dos efectos sobre el crecimiento y la desigualdad (Slag, Burger y Veenhoven, 2018). Por su parte, Tella, MacCulloch y Oswald (2003) utilizando datos de Estados Unidos y 12 países europeos, revelan que la evolución positiva de las variables macroeconómicas se asocia con la felicidad reportada. Japón también es un ejemplo de la asociación positiva entre ingresos y felicidad media (Stevenson y Wolfers, 2008).

Kahneman (2002) mostró con datos de Estados Unidos que el incremento del ingreso no se comporta directamente con variaciones positivas de la felicidad. Igualmente, en Taiwan y Malasia, Lim, Shaw y Liao (2017) no hallaron evidencia significativa. Gerstenblüth, Melgar y Rossi (2013) mencionan que el ingreso tiene poca significancia por sí solo cuando se le relaciona con los determinantes de la felicidad de los individuos. En efecto, según Frey y Stutzer (2002) en los países desarrollados el incremento en la renta media por causa de un mayor crecimiento económico, no ha traído mayores niveles de felicidad media en los últimos 50 años. Por este motivo se ha buscado adicionar variables de control para mejorar los resultados. Krueger y Schkade (2008) infieren que un grupo amplio de características internas y externas de los individuos pueden correlacionarse con el bienestar subjetivo tales pueden ser el empleo, su seguridad o su salud.

Layard, del Rey y Ramírez (2005) analizaron en la World Values Survey, 50 países en cuatro años. En el modelo logit utilizado los autores encontraron que los europeos eran más felices que los estadounidenses porque tienen una facilidad mayor para compartir socialmente, pues trabajan menos horas. Entre las variables de control estuvieron: las relaciones familiares, situación financiera, relación con el trabajo, comunidad y amigos, su percepción de la libertad, salud y valores personales. 
En la evidencia para Latinoamérica, Godoy-Jaramillo (2019) estudia los determinantes de la felicidad en Ecuador con los microdatos de la encuesta Latino barómetro de 2017. Los resultados del modelo ordinal utilizado muestran que el principal determinante de la felicidad es el ingreso, por lo cual propone una mejor distribución de los ingresos a través de un sistema impositivo más progresivo. En Colombia, se han hecho investigaciones empíricas relacionadas con la economía de la felicidad (Londoño-Vélez, 2011). Y entre las cuantitativas que se relacionan con la hipótesis planteada en la presente investigación, Pinzón-Gutiérrez (2017) estudia la pobreza relativa con base en los microdatos de la Encuesta Nacional de Calidad de Vida del Departamento Administrativo Nacional de Estadísticas (DANE, 2011). Con un modelo logit evalúan los determinantes de la pobreza subjetiva. Los resultados muestran que el ingreso tiene una relevancia significativa en la percepción de la pobreza, pero aspectos como tener una buena nutrición, la percepción de seguridad y tener buenos vínculos sociales también fueron aspectos relevantes. Estos resultados van más allá de los encontrados en Ecuador por Godoy (2019), cuyo principal determinante es el ingreso.

En línea con los resultados de este trabajo están los de Cruz y Torres (2006) que aplicaron un modelo probabilístico de elección discreta en el que la salud tiene una relación positiva frente a la percepción de satisfacción. El estar desempleado disminuye las probabilidades de responder que sus condiciones son buenas o muy buenas en $4 \%$ y 0,5\%, respectivamente. La relación edad y felicidad tiene una relación de $\mathrm{U}$ inversa, donde su inflexión ocurre alrededor de los 50 años. El capital humano también ofrece una relación positiva con la felicidad dada su relación con las condiciones de vida. Por cada año más de educación, la probabilidad de estar satisfecho con sus condiciones de vida se incrementa en $0,07 \%$.

Con base en la anterior revisión, se aprecia la necesidad de aplicar estudios cuantitativos que abarquen la felicidad desde una perspectiva más allá del ingreso monetario para validar o no la paradoja de Easterlin. 


\section{Metodología}

\section{Datos}

Se utilizaron microdatos de la Encuesta de Calidad de Vida (ECV) de 2017 realizada en Colombia por el DANE (2017). En esta se indaga por las condiciones físicas de los individuos como su vivienda, adquisición de bienes tamaño del hogar, etc. Igualmente, se obtuvo una visión de las características socioeconómicas de los individuos (edad, sexo, nivel educativo e ingreso, entre otras). Finalmente, se indagó por las percepciones del individuo como su satisfacción por el empleo, la salud, la seguridad y su vida. Su cobertura es de los 32 departamentos del país. Para esta investigación se utilizaron los datos de las personas que habían reportado todas las respuestas a la encuesta relacionadas con el módulo de percepción con un total de 15.2 millones de personas.

Tabla 1. Promedio de variables seleccionadas

\begin{tabular}{c|c|c|c|c|c|c|} 
Sexo & Edad & $\begin{array}{c}\text { Desvia- } \\
\text { ción }\end{array}$ & $\begin{array}{c}\text { Ingreso } \\
\text { per cápita }\end{array}$ & $\begin{array}{c}\text { Desvia- } \\
\text { ción }\end{array}$ & $\begin{array}{c}\text { Tamaño } \\
\text { hogar }\end{array}$ & Número \\
\hline Hombre & 46 & 15.7 & $1,057,284$ & $2,004,597$ & 3.34 & $9,581,092$ \\
\hline Mujer & 49 & 16.8 & 984,622 & $2,105,796$ & 3.07 & $5,618,847$ \\
\hline Total & 47 & 16.1 & $1,030,424$ & $2,042,899$ & 3.24 & $15,199,939$ \\
\hline
\end{tabular}

Fuente: elaboración propia con base en la ECV del DANE (2017)

Según la ECV utilizada en la investigación, los hombres tienen una media de 46 años frente a los 49 de las mujeres. El ingreso medio por hogar es de 1 millón de pesos con un tamaño medio de hogar de 3.24 personas.

Para esta investigación la variable de interés son los niveles de felicidad auto reportados por el individuo. Esta se reporta en niveles de 0 a 10 para su implementación en este trabajo; estos niveles se resumen en cinco niveles de 0 a 5. Esto facilitó la optimización del modelo y la interpretación de los resultados. Los diferentes niveles medios de felicidad se distribuyen así según los niveles de ingreso. 
Tabla 2. Porcentaje de la población en cada nivel de felicidad y cuantil de ingreso

\begin{tabular}{|c|c|c|c|c|c|c|}
\hline \multirow{2}{*}{$\begin{array}{l}\text { Nivel de } \\
\text { felicidad }\end{array}$} & \multicolumn{5}{|c|}{ Cuantil de ingreso } & \multirow{2}{*}{ Total } \\
\hline & 1 & 2 & 3 & 4 & 5 & \\
\hline 0 & 3.42 & 2.11 & 1.83 & 2.01 & 1.14 & 1.93 \\
\hline 1 & 2.50 & 1.79 & 1.48 & 1.02 & 0.99 & 1.43 \\
\hline 2 & 5.72 & 4.18 & 3.17 & 2.93 & 2.05 & 3.30 \\
\hline 3 & 15.64 & 14.46 & 13.02 & 10.44 & 8.21 & 11.64 \\
\hline 4 & 37.29 & 38.52 & 38.71 & 38.38 & 37.07 & 37.94 \\
\hline 5 & 35.41 & 38.94 & 41.80 & 45.22 & 50.54 & 43.76 \\
\hline
\end{tabular}

Fuente: elaboración propia con base en la ECV del DANE (2017)

El análisis descriptivo permite observar que el $80 \%$ de la población se encuentra en los niveles más altos de felicidad observada (4 y 5). En el caso de los cuantiles altos (4 y 5) el 45\% y 50\% de la población se encuentra en el nivel más alto de felicidad. La naturaleza de las variables identificadas en la ECV justifica la utilización de modelos que se adapten al conjunto de variables explicativas.

Ahora, el modelo utilizado para encontrar el efecto del ingreso sobre el nivel de felicidad de los colombianos es de tipo ordinal probabilístico. La variable objetivo es de tipo secuencial ordinal como se muestra en la figura 1.

Figura 1. Distribución de estudiantes para el estilo pragmático

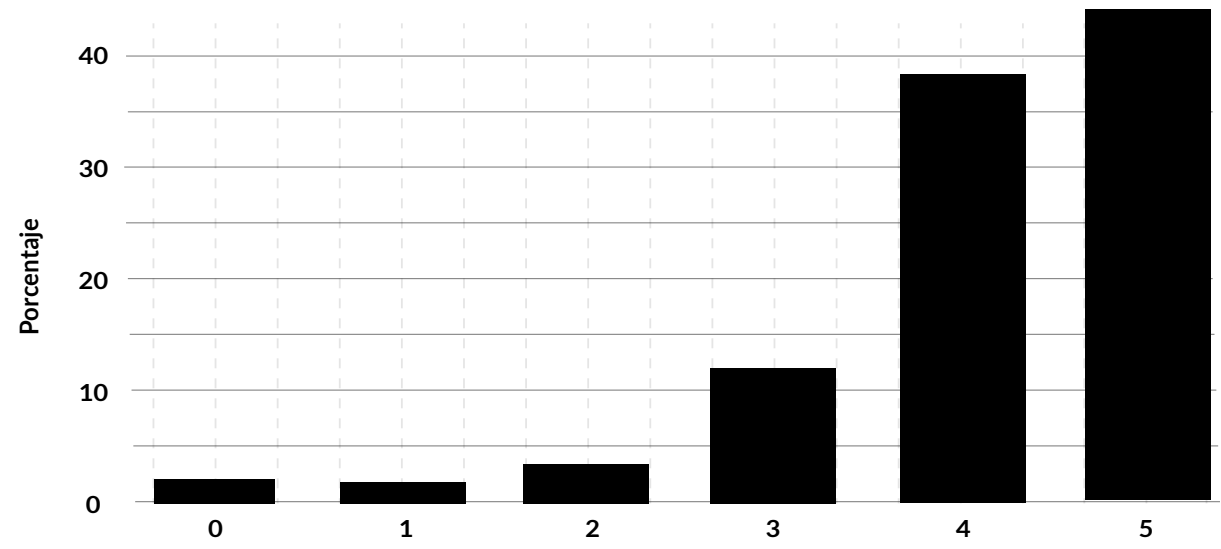

Fuente: elaboración propia 
Los modelos ordinales son un tipo de estimación probabilística que se basan en la existencia de una variable latente continua ( $\left.\mathrm{Y}^{*}\right)$ que no es observable, pero que condiciona la variable dependiente observada (Y) (Williams, 2006). Dicho proceso también supone que $\mathrm{Y}$ es discreta y está dividida la naturaleza de $\mathrm{Y}^{*}$ en puntos de cortes que tienen una diferenciación estadísticamente significativa entre sí. Se puede representar lo anterior así:

$$
\begin{gathered}
Y_{1}=1 \text { si } Y^{*} \leq \kappa_{1} \\
Y_{2}=1 \text { si } \kappa_{1} \geq Y^{*} \leq \kappa_{2} \\
Y_{3}=1 \text { si } Y^{*} \geq \kappa_{2}
\end{gathered}
$$

Para la estimación se partió de un modelo de regresión lineal sobre la variable $\mathrm{Y}^{*}$ :

$$
Y^{*}=\sum_{k=1}^{K} B_{k} X_{K i}+\varepsilon_{i} S i \rightarrow Z_{i}=\sum_{k=1}^{K} B_{k} X_{K i} \Rightarrow Y^{*}=Z_{i}+\varepsilon_{i}
$$

Donde i son cada una de las observaciones dentro de la base de datos, $K$ es el número de variables de control y $\varepsilon$ es el error. El modelo ordinal probabilístico requiere de una transformación del valor esperado de la ecuación (1) de esta manera:

$$
Z_{i}=\sum_{k=1}^{K} B_{k} X_{K i}=E\left(Y^{*}\right)=P\left(\kappa_{i-1} \geq Y^{*} \leq \kappa_{i}\right)
$$

La función de $Z_{i}$ toma la forma de una curva logística expresado por una ecuación de la forma:

$$
P\left(\kappa_{i-1} \geq Y^{*} \leq \kappa_{i}\right)=\frac{1}{1+\exp \left(Z_{i}-\kappa_{i}\right)}-\frac{1}{1+\exp \left(Z_{i}-\kappa_{i-1}\right)}
$$

El impacto de los coeficientes obtenidos dependerá de la influencia del número de datos que se ubiquen dentro de cada uno de las categorías de la variable objetivo. La ecuación a calcular dentro de esta investigación utilizó como dependiente una variable ordinal con seis intervalos en función de un grupo de 
variables de control que se relacionan con las características socioeconómicas del individuo:

Las variables de control fueron:

- Cuantil de ingreso: indica en que tramo del ingreso per cápita se encuentra el entrevistado. En este sentido se divide la distribución del ingreso en cinco intervalos y se asigna una variable categórica que identifica a cada individuo dentro de cada grupo.

- Indicador salud: es una escala de uno a cinco, los individuos evalúan el grado de conformidad con su estado de salud.

- Indicador trabajo: es una escala de uno a cinco, los individuos evalúan el grado de satisfacción con su trabajo.

- Percepción de seguridad: es una escala de uno a cinco, los individuos evalúan el grado en que se sienten seguros en su comunidad.

- Tipo de vivienda: si el hogar del entrevistado habita una vivienda de tipo casa, apartamento o habitación.

- Edad: Cuantos años cumplidos tiene el entrevistado.

- Departamento: Donde se localiza el hogar del entrevistado.

- Sexo del entrevistado

- Personas en el hogar: Número de personas en el hogar.

Nivel educativo: máximo nivel alcanzado en el sistema de educación colombiano

La variable independiente es categórica (el ingreso) que busca probar las hipótesis de la paradoja de Easterlin.

$$
P\left(\kappa_{i-1} \geq Y^{*} \leq \kappa_{i}\right)=f\left(Z_{i}\right)=\sum_{k=1}^{K} B_{k} X_{K i}
$$


Siendo $X_{K}$ el vector de variables independientes. Los coeficientes $\left(B_{k}\right)$ muestran el cambio en logaritmo de las probabilidades sobre $\mathrm{Y}$ frente a un cambio en la unidad de medida de la variable $\mathrm{X}$ asociada al coeficiente respectivo. Para obtener los efectos marginales se debe utilizar una función inversa logística de la ecuación 3. El software para los cálculos anteriormente señalados es StataCorp (2013).

\section{Resultados}

La tabla 4 muestra los resultados de las estimaciones del modelo ordinal enseñando los efectos marginales de cada una de las variables independientes sobre la probabilidad de encontrarse en el intervalo más alto de felicidad reportado (PENF). En términos del ajuste global el modelo resulta aceptable considerando que todas las variables introducidas presentaron niveles de significancia estadística del 5\% y según el $\mathrm{R}^{2}$ el modelo consigue explicar un 10\% de la varianza de la variable dependiente.

Tabla 3. Resultados de las estimaciones del modelo ordinal. Colombia 2017

\begin{tabular}{|c|c|c|c|c|}
\hline Variables & $\begin{array}{c}\text { Característica } \\
\text { Evaluada }\end{array}$ & Efecto Marginal & Error & $\begin{array}{c}\text { Carac- } \\
\text { terística } \\
\text { base }\end{array}$ \\
\hline \multirow{4}{*}{ Cuantil de ingreso } & 2 & $0.0456^{* * *}$ & (0.00109) & \multirow{4}{*}{1} \\
\hline & 3 & $0.0855^{* * *}$ & $(0.00110)$ & \\
\hline & 4 & $0.134^{* * *}$ & $(0.00113)$ & \\
\hline & 5 & $0.207^{* * *}$ & $(0.00127)$ & \\
\hline \multirow{5}{*}{ Indicador salud } & 1 & $0.109^{* * *}$ & $(0.00545)$ & \multirow{5}{*}{0} \\
\hline & 2 & $0.319^{* * *}$ & (0.00476) & \\
\hline & 3 & $0.499^{* * *}$ & $(0.00462)$ & \\
\hline & 4 & $0.712^{* * *}$ & $(0.00460)$ & \\
\hline & 5 & $1.115^{* * *}$ & $(0.00463)$ & \\
\hline \multirow{5}{*}{ Indicador trabajo } & 1 & $0.0505^{* * *}$ & $(0.00292)$ & \multirow{5}{*}{0} \\
\hline & 2 & $-0.0305^{* * *}$ & $(0.00247)$ & \\
\hline & 3 & $0.114^{* * *}$ & $(0.00221)$ & \\
\hline & 4 & $0.391^{* * *}$ & $(0.00218)$ & \\
\hline & 5 & $0.862^{* * *}$ & $(0.00225)$ & \\
\hline
\end{tabular}




\begin{tabular}{|c|c|c|c|c|}
\hline Variables & $\begin{array}{c}\text { Característica } \\
\text { Evaluada }\end{array}$ & Efecto Marginal & Error & $\begin{array}{c}\text { Carac- } \\
\text { terística } \\
\text { base }\end{array}$ \\
\hline \multirow{5}{*}{$\begin{array}{l}\text { Percepción de } \\
\text { seguridad }\end{array}$} & 1 & $0.0782^{* * *}$ & $(0.00345)$ & \multirow{5}{*}{0} \\
\hline & 2 & $0.0324^{* * *}$ & $(0.00295)$ & \\
\hline & 3 & $0.111^{* * *}$ & $(0.00277)$ & \\
\hline & 4 & $0.176^{* * *}$ & $(0.00273)$ & \\
\hline & 5 & $0.432^{* * *}$ & $(0.00278)$ & \\
\hline \multirow{4}{*}{ Tipo de vivienda } & Apartamento & $0.0212^{* * *}$ & $(0.000704)$ & \multirow{4}{*}{ Casa } \\
\hline & Cuarto(s) & $-0.0712^{* * *}$ & (0.00193) & \\
\hline & Indígena & $-0.113^{* * *}$ & $(0.00375)$ & \\
\hline & Otros & $-0.197^{* * *}$ & $(0.00978)$ & \\
\hline $\mathrm{Edad}^{\wedge} 2$ & & $0.0455^{* * *}$ & $(0.000500)$ & Continua \\
\hline \multirow{22}{*}{ Departamento } & Atlántico & $-0.0507^{* * *}$ & $(0.00163)$ & \multirow{22}{*}{ Antioquia } \\
\hline & Bogotá, D.C. & $-0.0995^{* * *}$ & $(0.00111)$ & \\
\hline & Bolívar & $-0.106^{* * *}$ & $(0.00164)$ & \\
\hline & Boyacá & $0.0484^{* * *}$ & $(0.00195)$ & \\
\hline & Caldas & $-0.0114^{* * *}$ & $(0.00264)$ & \\
\hline & Caquetá & $0.0323^{* * *}$ & (0.00271) & \\
\hline & Cauca & $-0.0881^{* * *}$ & (0.00184) & \\
\hline & Cesar & $0.148^{* * *}$ & $(0.00261)$ & \\
\hline & Córdoba & $0.144^{* * *}$ & $(0.00195)$ & \\
\hline & Cundinamarca & $-0.0923^{* * *}$ & $(0.00141)$ & \\
\hline & Chocó & $0.0358^{* * *}$ & $(0.00307)$ & \\
\hline & Huila & $0.131^{* * *}$ & $(0.00216)$ & \\
\hline & La Guajira & $-0.00775^{* * *}$ & $(0.00267)$ & \\
\hline & Magdalena & $0.0980^{* * *}$ & $(0.00259)$ & \\
\hline & Meta & $0.0297^{* * *}$ & $(0.00220)$ & \\
\hline & Nariño & $-0.0748^{* * *}$ & $(0.00177)$ & \\
\hline & $\begin{array}{c}\text { Norte de Santan- } \\
\text { der }\end{array}$ & $0.0967^{* * *}$ & $(0.00210)$ & \\
\hline & Quindío & $0.0101^{* * *}$ & (0.00288) & \\
\hline & Risaralda & $-0.0233^{* * *}$ & $(0.00240)$ & \\
\hline & Santander & $0.0904^{* * *}$ & $(0.00168)$ & \\
\hline & Sucre & $0.0878^{* * *}$ & $(0.00273)$ & \\
\hline & Tolima & $-0.0214^{* * *}$ & $(0.00200)$ & \\
\hline
\end{tabular}




\begin{tabular}{|c|c|c|c|c|}
\hline Variables & $\begin{array}{l}\text { Característica } \\
\text { Evaluada }\end{array}$ & Efecto Marginal & Error & $\begin{array}{c}\text { Carac- } \\
\text { terística } \\
\text { base }\end{array}$ \\
\hline \multirow{8}{*}{ Departamento } & Valle del Cauca & $0.0235^{* * *}$ & (0.00135) & \multirow{8}{*}{ Antioquia } \\
\hline & Arauca & $0.117^{* * *}$ & (0.00383) & \\
\hline & Casanare & $0.127^{* * *}$ & $(0.00382)$ & \\
\hline & Putumayo & $-0.116^{* * *}$ & $(0.00280)$ & \\
\hline & $\begin{array}{c}\text { Archipiélago de } \\
\text { San Andrés }\end{array}$ & $-0.332^{* * *}$ & (0.00561) & \\
\hline & Amazonas & $0.0757^{* * *}$ & $(0.00675)$ & \\
\hline & Guainía & $-0.0507^{* * *}$ & (0.00946) & \\
\hline & Guaviare & $-0.0280^{* * *}$ & $(0.00564)$ & \\
\hline Sexo & Mujer & $-0.0425^{* * *}$ & $(0.000635)$ & Hombre \\
\hline \multirow{4}{*}{ Personas en el hogar } & 2 & $0.0789^{* * *}$ & (0.00102) & \multirow{4}{*}{1} \\
\hline & 3 & $0.0571^{* * *}$ & $(0.00102)$ & \\
\hline & 4 & $0.102^{* * *}$ & $(0.00108)$ & \\
\hline & Más de 5 & $0.0649^{* * *}$ & $(0.00112)$ & \\
\hline \multirow{7}{*}{ Nivel educativo } & Preescolar & $-0.160^{* * *}$ & (0.0110) & \multirow{7}{*}{ Ninguno } \\
\hline & Primaria & $-0.0717^{* * *}$ & $(0.00132)$ & \\
\hline & Secundaria & $-0.0450^{* * *}$ & $(0.00153)$ & \\
\hline & Media & $-0.0620^{* * *}$ & $(0.00146)$ & \\
\hline & Técnico con título & $-0.0255^{* * *}$ & $(0.00185)$ & \\
\hline & $\begin{array}{c}\text { Universitario con } \\
\text { título } \\
\end{array}$ & $-0.0686^{* * *}$ & (0.00173) & \\
\hline & Posgrado con título & $-0.0677^{* * *}$ & $(0.00211)$ & \\
\hline \multicolumn{2}{|c|}{ Constante corte 1} & $0.693^{* * *}$ & (0.00484) & \\
\hline \multicolumn{2}{|c|}{ Constante corte 2} & $0.948^{* * *}$ & $(0.00482)$ & \\
\hline \multicolumn{2}{|c|}{ Constante corte 3} & $1.316^{* * *}$ & $(0.00484)$ & \\
\hline \multicolumn{2}{|c|}{ Constante corte 4} & $2.004^{* * *}$ & $(0.00487)$ & \\
\hline \multicolumn{2}{|c|}{ Constante corte 5} & $3.222^{* * *}$ & (0.00493) & \\
\hline
\end{tabular}

\begin{tabular}{c|c|}
\hline Observaciones & $14,724,495$ \\
\hline R2 & 0.09955 \\
\hline
\end{tabular}

Fuente: elaboración propia

Errores estándares entre paréntesis. $\left.\right|^{* * *} p<0.01,{ }^{* *} p<0.05,{ }^{*} p<0.1$ 
Para la interpretación de los resultados, el efecto marginal en las variables continuas equivale al porcentaje en el que se incrementa la probabilidad de ubicarse, dado un cambio del $1 \%$ en la variable independiente de análisis. Por ejemplo, un incremento del cuadrado de la edad del individuo, afecta la probabilidad de ser muy feliz en un 0,04\%. Para las variables categóricas, el efecto marginal mide el cambio en la probabilidad de estar en el nivel más alto de felicidad en comparación con la característica base; por ejemplo, las personas que habitan en apartamentos tienen un $0,02 \%$ más de probabilidad de encontrarse en el nivel 5 de felicidad (PENF) frente a los que viven en casa.

Frente a la hipótesis principal de este trabajo y en línea con la evidencia internacional, los individuos con mayores ingresos muestran en promedio niveles de felicidad superiores. Las personas en el cuantil 5 de ingreso son un $0,2 \%$ más probables de estar en PENF. Según estos mismos datos, la hipótesis de Kahneman (2002) que hasta cierto punto la riqueza incrementa la felicidad y luego se mantiene, no se aplicaría pues el cambio marginal entre cuantiles se mantiene constante. Pasando a la segunda hipótesis, sobre la importancia del ingreso, se compara el efecto del mismo frente a las otras condiciones que percibe el individuo. La figura 1 muestra que para los individuos que se ubican en el nivel 5 de felicidad, los aspectos de un tener buen trabajo, seguridad y salud pesan más que estar en el cuantil 5 de ingreso. Por lo tanto, se valida la paradoja de Easterlin, donde se dice que el dinero no logra la felicidad en su totalidad, sino que se debe complementar con otros aspectos de la vida.

Para las variables de control, los hombres declaran estar más felices que las mujeres, en línea con los resultados de la evidencia internacional (Lykken y Tellegen, 1996). El capital social también se asocia positivamente con la percepción de los individuos, pues los hogares unipersonales tienen una probabilidad de -0,07\% de PENF. En este mismo sentido, las personas con mayores niveles de educación tienen -0.06\% de PENF frente a los que no tienen ninguno. Igualmente, se muestran diferencias geográficas significativas. 


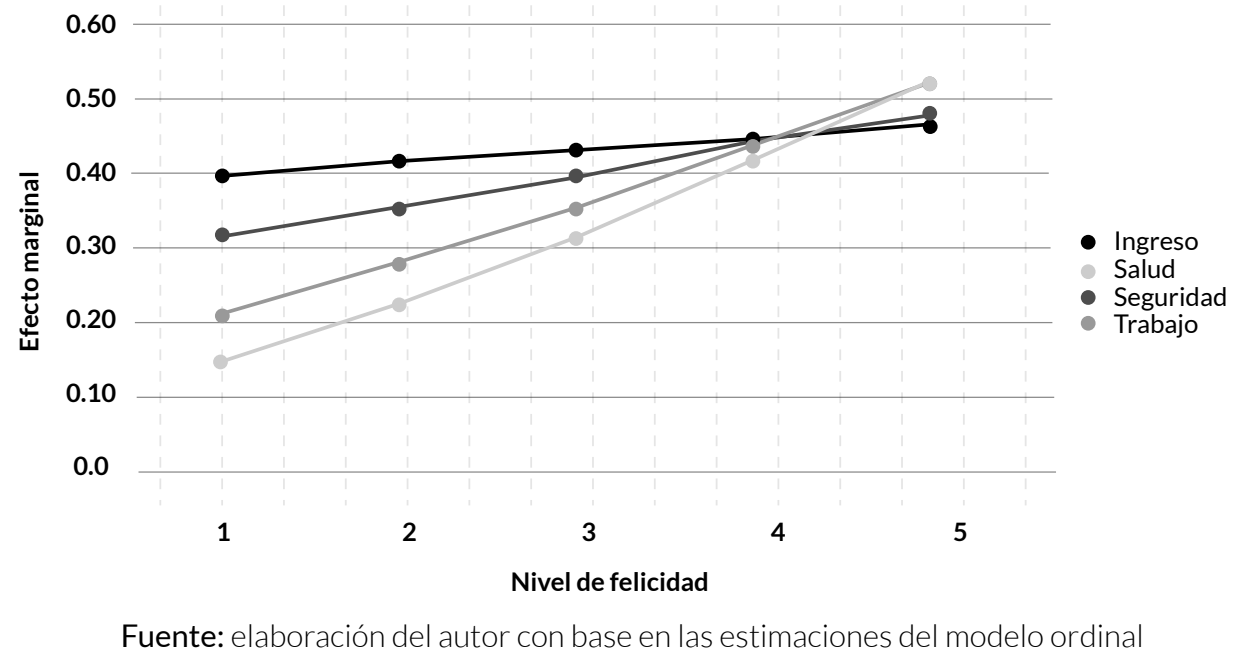

\section{Conclusiones}

La felicidad es un fenómeno complejo que se desarrolla en el ámbito individual y social; sin embargo, según Veenhoven (2007), la felicidad de los individuos conlleva información importante sobre la calidad de los modelos de desarrollo impulsados por los gobiernos. Por esto, la llamada "economía de la felicidad" ha querido investigar los determinantes de la misma en términos del plano material de los individuos. Dentro de estos estudios ha habido interés sobre el estudio de la paradoja de Easterlin; esta dice que la relación entre los niveles de ingreso y la felicidad no es proporcionalmente positiva, es decir, que aumentos del ingreso no se correlacionan con los cambios en la felicidad, en consecuencia, hay otros factores más determinantes que ponderan los individuos para su percepción de calidad de vida.

Este trabajo exploró con base en los datos de la Encuesta de Calidad de Vida 2017 en Colombia esta paradoja, esto es, qué el papel juega el ingreso en el bienestar de los colombianos. El modelo ordinal propuesto evidenció que el ingreso es una variable determinante de la felicidad, pero es secundaria frente a otras como la percepción de salud, seguridad y el trabajo; tales resultados están en línea con los planteados en la paradoja y en la evidencia internacional. Otro resultado de interés, es que el efecto marginal de cada cuantil de ingreso muestra que el efecto del ingreso sobre la felicidad es marginalmente lineal, es decir, que 
no se cumple la hipótesis de que, a partir de cierto umbral de ingreso, este ya no ejerce una influencia en la felicidad de las personas.

Los limitantes del presente estudio se basan en la fuente de información, dado que la felicidad revelada por los individuos es de tipo subjetivo, por lo tanto, puede estar influida por aspectos coyunturales al momento de la encuesta. Igualmente, las extensiones del presente estudio pueden estar enfocados al análisis longitudinal del individuo y cómo cambian sus percepciones frente a cambios de la política pública aplicada a su contexto individual, familiar y de riqueza.

\section{Referencias}

Borrero, S.; Escobar, A. B.; Cortés, A. M.; Maya, L. C. (2013). Poor and Distressed, but Happy: Situational and Cultural Moderators of the Relationship between Wealth and Happiness. Estudios Gerenciales, 29(126), 2-11. https://www.icesi.edu.co/revistas/index.php/estudios_gerenciales/ article/view/ 1592

Botello, H. A.; Ríos, G. (2014). Determinantes de la satisfacción en el empleo en el Área Metropolitana de Bucaramanga, 2008-2012. Libre Empresa, 11(1), 1325. https://doi.org/10.18041/1657-2815/libreempresa.2014v11n 1.3010

Cruz, J.; Torres, J. (2006). ¿De qué depende la satisfacción subjetiva de los colombianos? Cuadernos de Economía, 25(45), 131-154. https://revistas.unal.edu.co/index.php/ceconomia/article/view/552

Departamento Administrativo Nacional de Estadística, DANE. (2011). Encuesta nacional de calidad de vida (ECV). https://www.dane.gov.co/index.php/ estadisticas-por-tema/salud/calidad-de-vida-ecv/encuesta-nacional-decalidad-de-vida-2011

Departamento Administrativo Nacional de Estadística, DANE. (2017). Encuesta nacional de calidad de vida (ECV). https://www.dane.gov.co/index.php/ estadisticas-por-tema/salud/calidad-de-vida-ecv/encuesta-nacional-decalidad-de-vida-2011

Diener, E.; Diener, M.; Diener, C. (1995). Factors Predicting the Subjective Well-Being of Nations. Journal of Personality and Social Psychology, 69(5), 851-864. https://doi.org/10.1007/978-90-481-2352-0_3 
Easterlin, R. A. (1974). Does Economic Growth Improve the Human lot? Some Empirical Evidence. En Nations and Households in Economic Growth (pp. 89-125). Academic Press. https://doi.org/10.1016/b978-0-12-2050503.50008-7

Easterlin, R. A. (1995). Will Raising the Incomes of all Increase the Happiness of All? Journal of Economic Behavior E Organization, 27(1), 35-47. https://doi.org/10.1016/0167-2681(95)00003-b

Frank, D. H. (1985). The End of the Guide: Maimonides on the Best Life for Man. Judaism, 34(4), 485. https://doi.org/10.4135/9781526401632.n 1

Frey, B. S.; Stutzer, A. (2002). What can Economists Learn from Happiness Research? Journal of Economic literature, 40(2), 402-435. https://doi. org/10.1257/jel.40.2.402

Gerstenblüth, M.; Melgar, N.; Rossi, M. (2013). Ingreso y desigualdad. ¿Cómo afectan a la felicidad en América Latina? Cuadernos de Economía, 32(59), 163-178. http://www.scielo.org.co/scielo. php? script=sci_arttext\&pid=So $121-47722013000100008$

Godoy-Jaramillo, J. I. (2019). La felicidad en Ecuador: una aproximación a sus determinantes. (Tesis de pregrado). Universidad de la Loja. https://dspace.unl.edu.ec/jspui/handle/123456789/21818

Kahneman, D. (2002). Maps of Bounded Rationality: A Perspective on Intuitive Judgment and Choice. Nobel prize lecture, 8, 351-401. https://doi.org/10.1037/0003-066x.58.9.697

Krueger, A. B.; Schkade, D. A. (2008). The Reliability of Subjective WellBeing Measures. Journal of Public Economics, 92(8-9), 1833-1845. https://doi.org/10.3386/w 13027

Layard, R.; del Rey, V. E. G.; Ramírez, M. (2005). La felicidad: lecciones de una nueva ciencia. En Layard, Richard. Felicidad: Lecciones de una nueva ciencia (pp. 60-89). Taurus

Lim, H. E.; Shaw, D.; Liao, P. S. (2017). Revisiting the Income-happiness Paradox: The Case of Taiwan and Malaysia. Institutions and Economies, 4(9), 53-69. https://doi.org/10.2139/ssrn.2883618 
Londoño-Vélez, J. (2011). Movilidad social, preferencias redistributivas y felicidad en Colombia. Revista Desarrollo y Sociedad, 68, 171-212. https://doi.org/10.13043/dys.68.6

Lykken,D.; Tellegen,A.(1996).HappinessisaStochasticPhenomenon.Psychological Science, 7(3), 186-189. https://doi.org/10.1111/j.1467-9280.1996. tbo0355.x

Mikucka, M.; Sarracino, F.; Dubrow, J. K. (2017). When Does Economic Growth Improve Life Satisfaction? Multilevel Analysis of the Roles of Social Trust and Income Inequality in 46 Countries, 1981-2012. World Development, 93, 447-459. https://doi.org/10.1016/j.worlddev.2017.01.002

Oishi, S.; Kesebir, S. (2015). Income Inequality Explains Why Economic Growth Does not Always Translate to an Increase in Happiness. Psychological Science, 26(10), 1630-1638. https://doi.org/10.1177/0956797615596713

Pinzón-Gutiérrez, L. F. (2017). Factores asociados a la pobreza subjetiva en Colombia: un estudio desde el enfoque de las capacidades y la economía de la felicidad. Revista Desarrollo y Sociedad, 78, 11-57. https://doi.org/10.13043/dys.78.1

Reich, J.; Diener, E. (1994). The Road to Happiness. (Cover story). Psychology Today, 27(4), 32. https://doi.org/10.1057/9781137321534_2

Slag, M., Burger, M. J.; Veenhoven, R. (2019). Did the Easterlin Paradox Apply in South Korea between 1980 and 2015? A Case Study. International Review of Economics, 66(4), 325-351. https://doi.org/10.1007/s12232019-00325-w

Stevenson, B.; Wolfers, J. (2008). Economic Growth and Subjective Well-Being: Reassessing the Easterlin Paradox. National Bureau of Economic Research, No. w1 4282. https://doi.org/10.3386/w 14282

Tella, R. D.; MacCulloch, R. J.; Oswald, A. J. (2003). The Macroeconomics of Happiness. Review of Economics and Statistics, 85(4), 809-827.

Torrecilla, O. D. (2005). Clima organizacional y su relación con la productividad laboral [Documento de trabajo]. Facultad de Ciencias Políticas y sociales. 
Veenhoven, R. (2007). World Database of Happiness: Continuous Register of Scientific Research on Subjective Appreciation of Life. World Database of Happiness. https://www.researchgate.net/publication/228659294_ World_Database_of_Happiness_Continuous_register_of_research_on_ subjective_appreciation_of_life

Williams, R. (2006). Generalized Ordered Logit/ Partial Proportional Odds Models for Ordinal Dependent Variables. The Stata Journal 6(1), 58-82. https://doi.org/10.1177/1536867x0600600104

\section{Anexo}

Tabla 5. Matriz de correlaciones

\begin{tabular}{c|c|c|c|c|c|c|c|c|c} 
& Sexo & Cuantil & $\begin{array}{c}\text { Tipo } \\
\text { Vivienda }\end{array}$ & Edad & $\begin{array}{c}\text { indicador } \\
\text { salud }\end{array}$ & $\begin{array}{c}\text { indicador } \\
\text { trabajo }\end{array}$ & $\begin{array}{c}\text { indicador } \\
\text { seguridad }\end{array}$ & $\begin{array}{c}\text { \# per. } \\
\text { hogar }\end{array}$ & Educación \\
\hline Sexo & 1,00 & & & & & & & & \\
\hline Cuantil & $-0,03$ & 1,00 & & & & & & & \\
\hline $\begin{array}{c}\text { Tipo } \\
\text { Vivienda }\end{array}$ & 0,05 & 0,09 & 1,00 & & & & & & \\
\hline $\begin{array}{c}\text { Edad } \\
\text { indicador } \\
\text { salud }\end{array}$ & $-0,04$ & 0,08 & $-0,11$ & 1,00 & & & & & \\
\hline $\begin{array}{c}\text { indicador } \\
\text { trabajo }\end{array}$ & $-0,0,13$ & 0,22 & 0,02 & $-0,08$ & 0,39 & 1,00 & & & \\
\hline $\begin{array}{c}\text { indicador } \\
\text { seguridad }\end{array}$ & $-0,05$ & 0,03 & $-0,02$ & 0,00 & 0,29 & 0,30 & 1,00 & & \\
\hline $\begin{array}{c}\text { \# per. } \\
\text { hogar }\end{array}$ & $-0,09$ & $-0,32$ & $-0,09$ & $-0,14$ & 0,04 & 0,01 & $-0,02$ & 1,00 & \\
\hline Educación & 0,04 & 0,44 & 0,14 & $-0,20$ & 0,17 & 0,16 & $-0,02$ & $-0,06$ & 1,00 \\
\hline
\end{tabular}

Fuente: elaboración propia 


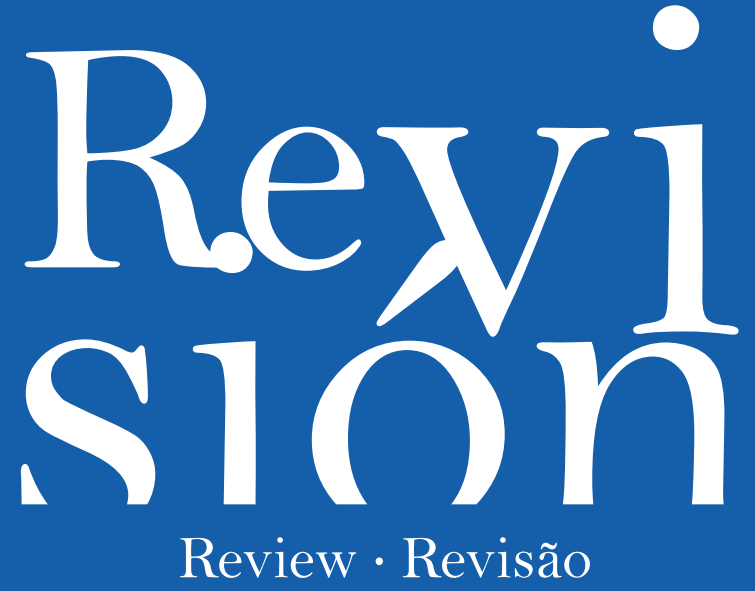


